\title{
A Case Report of a Misleading Mammary Tuberculosis in a Young Patient
}

\author{
Harou Karam, Billa Khalid, Boukhanni Lahcen, Asmouki Hamid, Soummani Abderraouf \\ Obstetrics and Gynecology Department, Mohammed VI University Hospital Center, Faculty of Medicine and Pharmacy, \\ Marrakech, Morocco \\ Email: dr.billa.khalid@gmail.com
}

How to cite this paper: Karam, H., Khalid, B., Lahcen, B., Hamid, A. and Abderraouf, S. (2019) A Case Report of a Misleading Mammary Tuberculosis in a Young Patient. Open Access Library Journal, 6: e5254. https://doi.org/10.4236/oalib.1105254

Received: February 15, 2019

Accepted: March 25, 2019

Published: March 28, 2019

Copyright $\odot 2019$ by author(s) and Open Access Library Inc.

This work is licensed under the Creative Commons Attribution International License (CC BY 4.0).

http://creativecommons.org/licenses/by/4.0/

\begin{abstract}
Mammary tuberculosis is a rare case of extrapulmonary tuberculosis. Through this case of primary mammary tuberculosis in a nulligest young patient, we will try to describe the clinical, paraclinical and therapeutic features of this entity.
\end{abstract}

\section{Subject Areas \\ Gynecology \& Obstetrics}

\section{Keywords}

Breast Lump, Mammary Tuberculosis, Tubercle Bacilli, Mycobacterium Tuberculosis

\section{Introduction}

Tuberculosis has traditionally been regarded as a pulmonary disorder. The elective extrapulmonary localizations are, in order of decreasing frequency: the ganglia, the peritoneum, the pericardium, the kidneys, the skin, the joints, the meninges and the bone [1]. Breast localization is very rare, accounting for less than $0.1 \%$ of cases [2] [3]. This location can be primitive or secondary to locoregional or disseminated tuberculosis, and it often poses diagnostic difficulties both clinically and paraclinically [1] [2].

In the absence of well-defined clinical features, the true nature of the disease remains obscure and it is often mistaken for carcinoma or pyogenic breast abscess [4].

The disease is eminently curable with the modern anti-tubercular chemotherapeutic drugs with surgery playing a role in the background only. 
The aim of our study is to make a clinical, paraclinical and therapeutic development on this rare pathology.

\section{Case Presentation}

A 32-year-old patient, a primary school teacher, nulliparous, presented to our department for bilateral mastalgia with left nipple discharge. The patient did not have a past medical history, neither a family tuberculosis cases.

Physical examination revealed a bilateral, tender, irregular nodules measuring approximately $2 \mathrm{~cm}$ in the upper-outer quadrant of the right and the left breast. There was no skin alteration. Whereas there were no palpable axillary or supraclavicular lymph nodes.

The mammography showed an architectural disorganization in the left breast (Figure 1).

Ultrasonography revealed a bilateral hypoechoicheterogeneous mass measuring $2 \mathrm{~cm}$ suggesting mastitis infection with breast abscess (Figure 2).

A bacteriological analysis of the nipple discharge showed the presence of staphylococcus aureus sensible to penicillin.

An antibiotic treatment (Penicillin M) was prescribed. Over the next two weeks her symptoms persisted despite antibiotic therapy. However, she presented intermittent fever, night sweats and spontaneous skin fistula of the left nodule.

A core needle biopsy of the left mass was performed and showed a granuloma with multinucleated giant cells, surrounding the epithelioid infected macrophages with caseous necrosis, leading to the diagnosis of breast tuberculosis (Figure 3).

The search of other localization of tuberculosis (thoracic radiography, cutaneous tuberculin test and microbiological examination of 3 days' sample of sputum) was negative.

The patient received a 6 months' medical treatment based on antituberculars including 2 months $\mathrm{RHZ}$ and 4 months $\mathrm{RH}$ according to the national protocol.

The evolution was good with a total regression of the lesion and without any recurrency in the last 12 months.

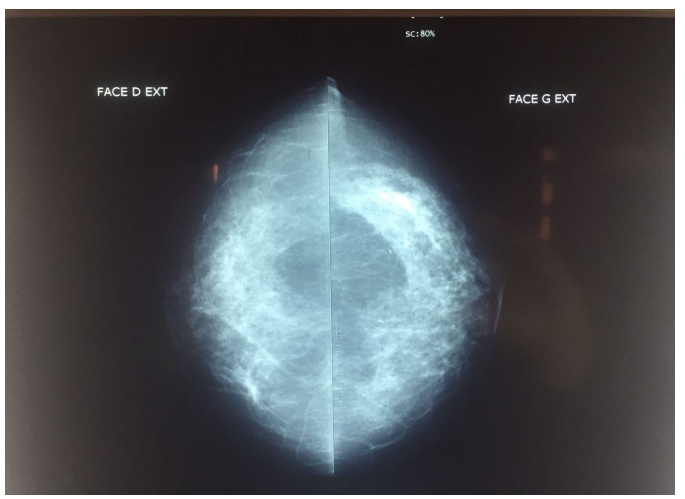

Figure 1. Mammography: architectural disorganization in the left breast. 

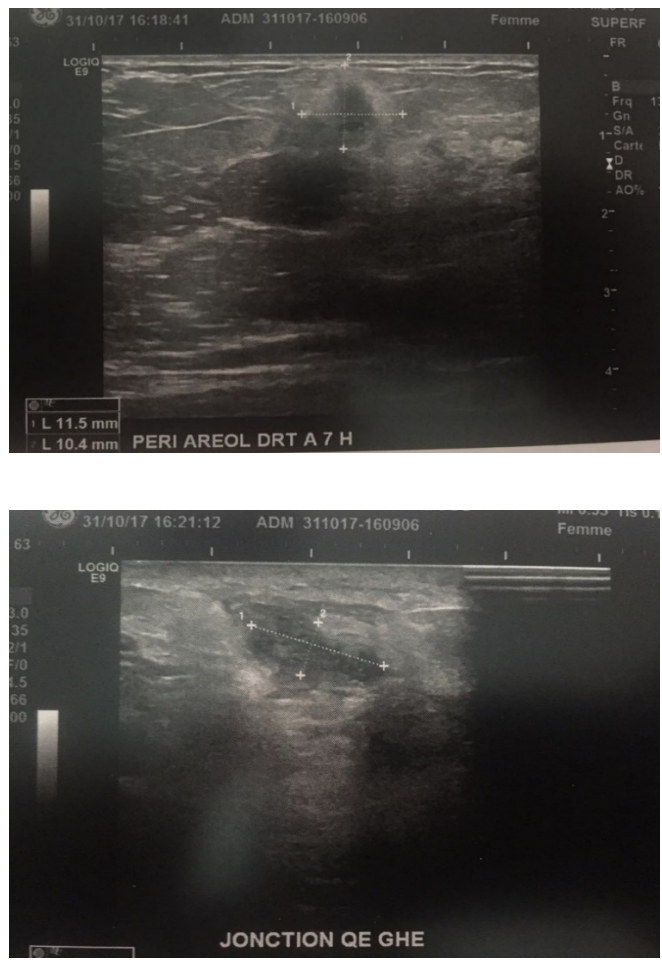

Figure 2. Hypoechoic heterogeneous mass measuring $2 \mathrm{~cm}$.
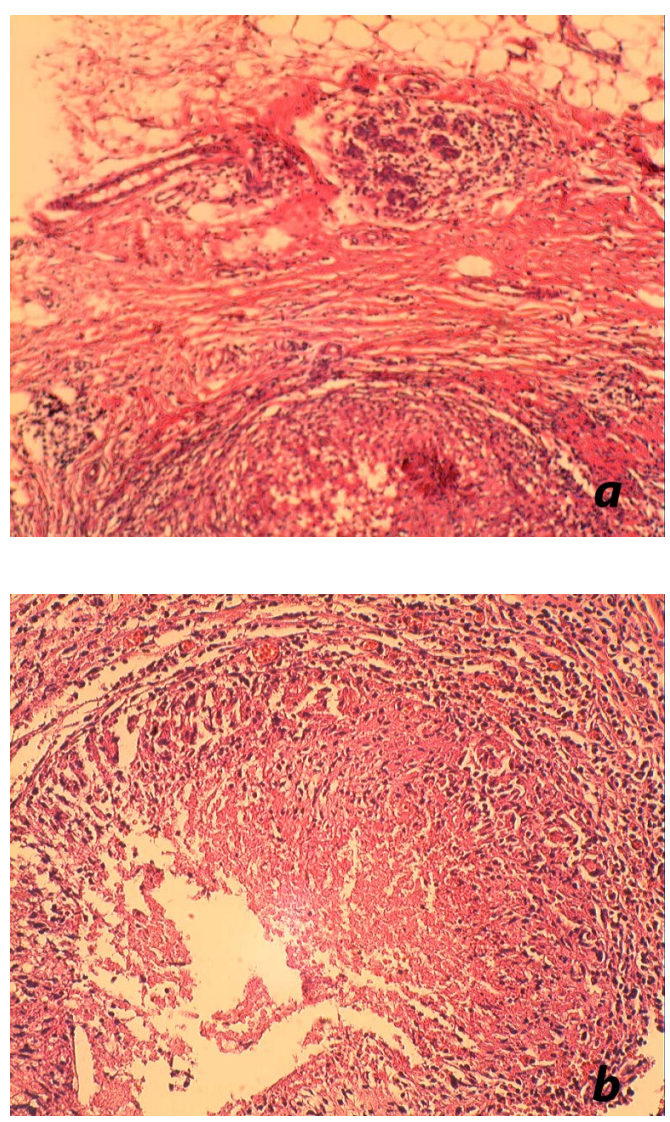

Figure 3. Epithelioid cell granulomas and caseous necrosis, lower (a) to higher (b) magnifications. 


\section{Discussion}

Breast tuberculosis was first described by Sir Astley Cooper in 1829, who called it "scrofulousswelling of the bosom". Breast tuberculosis is uncommon because of the resistance offered by the breast tissue to tubercle bacilli. It commonly affects multiparous females usually in the age group of $21-40$ years [5]. Our patient was a 32-year-oldnulliparous female.

Breast tuberculosis can be primary or secondary. Primary is when the tuberculous infection is confined to the breast and is extremely uncommon. It is secondary when there is a coexisting focus of tuberculosis somewhere else in the body, most commonly, pulmonary tuberculosis. Our patient was a case of primary tuberculosis of the breast [5].

The commonest clinical presentation is a painless mass that progresses with posterior cutaneous involvement and the formation of ulcers and fistula. The diffuse form is characterized by multiple drainage foci, with an important inflammatory process and axillary involvement. The less common sclerosing form occurs in elderly women and presents a higher degree of fibrosis, with nipple retraction and suppuration [6].

Although our patient didn't have any past history and the clinical presentation was bilateral.

The breast tuberculosis can mimic breast carcinoma, with irregular border, fixed to either the skin or the muscle or even to the chest wall [7]. Fistula formation may occur, much as nipple or skin retraction, but breast discharge is uncommon. The lump may be followed by inflammation and abscess formation, skin ulceration and diffuse mastitis. Recurrent inflammation and abscess of the breast that do not respond to surgical drainage and standard antibiotic therapy in young women should raise suspicion. Constitutional symptoms like fever with evening rise, malaise, night sweats and weight loss are present in less than $20 \%$ of the cases [8]. In our case the evolution was marked by the nonresponse to antibiotic treatment and the appearance of night sweats and intermittent fever.

The principal differential diagnosis is breast carcinoma. Other diseases of the breast such as fatty necrosis, plasma cell mastitis, periareolar abscess, idiopathic granulomatous mastitis and infections like actinomycosis and blastomycosis are to be considered [9].

The gold standard in establishing the diagnosis is demonstration of the causative organism Mycobacterium tuberculosis in Ziehl-Neelsen stain or in culture but is difficult to demonstrate. Polymerase chain reaction as a diagnostic tool for breast tuberculosis is less often reported. However, PCR is by no means absolute in diagnosing tubercular infection as false negative results are still a probability [10]. Ultrasound is helpful for characterizing the ill-defined densities shown on mammography by exclusion of solid masses, but the findings of a hypoechoic lesion with heterogeneous internal echoes and irregular borders are not specific [11].

The fine needle and core needle biopsies are usually the first non-invasive 
methods of obtaining histological specimen. The presence of caseating granulomas, epithelioid cells and lymphohistiocitosis will likely point the diagnosis [12].

The curative treatment pf breast tuberculosis is based on combination of tuberculostatic drugs of isoniazid, rifampicin, pyrazinamide and ethambutol with a 6 months' regimen [10]. According to the Moroccan guidelines the regimen is 2 intensive months of RHZ then 4 months RH of maintenance.

Surgery is at least reserved to the resistant form or a complement for abscess drainage

\section{Conclusions}

The mammary tuberculosis is a very rare localization, even in the countries of endemic disease. The clinical and radiological signs are misleading, and raise a diagnosis problem in particular with the breast cancer. Due to the rarity of the pathology mentioned in the present report, it is necessary to investigate differential diagnoses.

The anatomopathological examination stays the main element for the diagnosis of certainty.

Tuberculosis represents a rare disease that should always be suspected when evaluating cases of breast lump in women of reproductive age group, with poor response to classical non-tuberculosis antibiotic treatment. Early diagnosis and prompt treatment with anti-tuberculous therapy reduce morbidity.

\section{Consent}

Informed consent was obtained from the patient to report the case.

\section{Conflicts of Interest}

The authors declare no conflicts of interest regarding the publication of this paper.

\section{References}

[1] Ben Hassouna, J., Gamoudi, A., Bouzaiene, H., Dhiab, T., Khomsi, F., et al. (2005) Mammary Tuberculosis: A Retrospective Study of 65 Cases. Gynécologie Obstétrique \& Fertilité, 33, 870-876. https://doi.org/10.1016/j.gyobfe.2005.08.020

[2] Fadaei-Araghi, M., Geranpayeh, L., Irani, S., Matloob, R. and Kuraki, S. (2008) Breast Tuberculosis: Report of Eight Cases. Archives of Iranian Medicine, 11, 463-465.

[3] Luh, S.P., Hsu, J.D., Lai, Y.S. and Chen, S.W. (2007) Primary Tuberculous Infection of Breast: Experiences of Surgical Resection for Aged Patients and Review of Literature. Journal of Zhejiang University Science B, 8, 580-583. https://doi.org/10.1631/jzus.2007.B0580

[4] Thyagarajan, M., Nallasivam, M. and Singh, B. (2017) Bacilli in the Breast. International Surgery Journal, 4, 3344-3346.

[5] Gupta, A., Gupta, M., Gupta, J. (2017) Unusual Case of Bilateral Tubercular Mastitis. Cureus, 9, e1383.

[6] Quilles, M.B., Balbo, P.A.M., Benez, M.F., Nicolellis, L.P., Barbosa, R.N.W., Siqueira, F.P.C. and Zutin, L.T.M. (2017) Abscess in Primary Tuberculosis of Breast: Case 
Report. Journal of Tuberculosis Research, 5, 161-167. https://doi.org/10.4236/jtr.2017.53018

[7] Teo, T.H., Ho, G.H., Chaturverdi, A. and Khoo, B.K. (2009) Tuberculosis of the Chest Wall: Unusual Presentation as a Breast Lump. Singapore Medical Journal, 50, e97-e99.

[8] Marinopoulos, S., Lourantou, D., Gatzionis, T., Dimitrakakis, C., Papaspyrou, I. and Antsaklis, A. (2012) Breast Tuberculosis: Diagnosis, Management and Treatment. International Journal of Surgery Case Reports, 3, 548-550. https://doi.org/10.1016/j.ijscr.2012.07.003

[9] Akcay, M.N., Saglam, L., Polat, P., Erdogan, F., Albayrak, Y. and Povoski, S.P. (2007) Mammary Tuberculosis-Importance of Recognition and Differentiation from That of a Breast Malignancy: Report of Three Cases and Review of the Literature. World Journal of Surgery and Oncology, 5, 67. https://doi.org/10.1186/1477-7819-5-67

[10] Mirsaeidi, S.M., Masjedi, M.R., Mansouri, S.D. and Velayati, A.A. (2007) Tuberculosis of the Breast: Report of 4 Clinical Cases and Literature Review. Eastern Mediterranean Health Journal, 13, 670-676.

[11] Rahul, K., Silpa, K., Santosh, K. and Rajiv, G. (2017) Tuberculosis of Breast: A Rare Presentation. Journal of Case Reports, 7, 127-129.

[12] Lee, C.H. and Sharif, S.Z. (2016) Primary Breast Tuberculosis in a Patient with Known Invasive Breast Carcinoma: A Cas Report. Medical Journal of Malaysia, 71, 149-151. 